\title{
CONCEPTO DE ADHERENCIA PREVENTIVA EN EL ÁMBITO DE LAS ADICCIONES
}

\section{CONCEPT OF PREVENTIVE ADHERENCE IN THE FIELD OF ADDICTIONS}

\author{
Jose A. García del Castillo ${ }^{1}$ Álvaro García del Castillo-López y Carmen López-Sánchez ${ }^{2}$ \\ ${ }^{1}$ Universidad Miguel Hernández \\ ${ }^{2}$ Universidad de Alicante
}

\section{Abstract}

Even though several decades developing the prevention in the field of addictions, nowadays we still configuring its conceptual framework. In this work we pretend to take a step forwards on that configuration trying to specify in a theoretical way the adherence process in the preventive field. Starting from the theoretical case in the relation of the adherence with different basic psychological processes, the structure of the preventive adherence is shown through both different models and psychosocial theories wich have confirmed somehow its influence over adherence behaviour. Gathering the advances produced in several contexts where adherence processes are included, its development is proposed from the Social Cognitive Theory and the Transtheoretical Model. Finally several aspects related to the theoretical approach and its practicals implications are discussed.

Keywords: Preventive adherence, addictions, prevention programs, psychosocial theories

\section{Resumen}

A pesar de llevar varias décadas desarrollando el campo de la prevención de las adicciones, a día de hoy aún seguimos configurando su marco conceptual. En este trabajo se pretende dar un paso más en su construcción intentando concretar a nivel teórico el proceso de adherencia en el ámbito preventivo. Partiendo del supuesto teórico en la relación del concepto de adherencia con diferentes procesos psicológicos básicos, se plantea la estructura de la adherencia preventiva desde diferentes modelos y teorías psicosociales que de alguna forma han confirmado su influencia sobre el comportamiento de adherencia. Recogiendo los avances producidos en distintos contextos en los que se incluyen procesos de adherencia, se propone su desarrollo desde la Teoría Social Cognitiva y el Modelo Transteórico. Finalmente se discuten distintos aspectos relacionados con el planteamiento teórico y sus implicaciones prácticas.

Palabras clave: Adherencia preventiva, adicciones, programas de prevención, teorías psicosociales. 
Después de varias décadas de avance y desarrollo de la prevención de adicciones, seguimos construyendo lo que sería su marco teórico o conceptual, partiendo de la base de que se nutre de diferentes disciplinas consolidadas, pero carece de su propio ámbito de referencia. Paulatinamente se va perfilando la fundamentación en los que apoya sus principios y objetivos, como la percepción de riesgo (Bejarano, Ahumada, Sánchez, Nora, Hynes y Cumsille, 2011; García del Castillo, 2012; García del Castillo, López-Sánchez y García del Castillo-López, 2014; Gil, González y Meneses, 2010; Uribe, Verdugo y Salinas, 2011; Zimmermann, 2010), la información (García del Castillo, López-Sánchez, García del Castillo-López y Dias, 2014; Ordoñana y Gómez, 2002; Rendón, 2005; Ying, Jisoo, Lewis y Martínez, 2014), las técnicas y herramientas de intervención (Boustani, Frazier, Becker, Bechor, Dinizulu, Hedemann, Ogle y Pasalich, 2014; Gázquez, García del Castillo y Espada, 2011; Valentine y Dejong, 2013), la metodología y la evaluación (Ariza, Villalbí, Sánchez-Martínez y Nebot, 2011; García del Castillo, 2011; Gázquez, García del Castillo y Ruiz, 2011; Jiménez, Antolín-Suárez, Oliva, Hidalgo, Jiménez-Iglesias, Lorence, Moreno y Ramos, 2014; Holder, 2009; Londoño y Vinaccia, 2005), y las teorías y modelos (Becoña, 1999; García del CastilloLópez, García del Castillo y Marzo, 2012; Grant, Potenza, Weinstein y Gorelick, 2010; Pons, 2008; Olivar, 2011; Solinas, Thiriet, Chauvet y Jaber, 2010).

En los programas preventivos cualesquiera de estos conceptos, principios, modelos o teorías, juega un papel primordial en la efectividad y la eficiencia final. Un programa se construye conjugando la interacción de sus componentes y teniendo en cuenta que todos los elementos estén suficientemente contrastados y probados previamente en el contexto donde se van a aplicar. Que no se consigan los resultados esperados se le puede atribuir, grosso modo, a diferentes cuestiones:

- $\quad$ Errores en el diseño

- Problemas con la metodología

- $\quad$ Errores en la aplicación de modelos o teorías

- Problemas en las estrategias de intervención

- Fallos en el manejo de la información

- Baja adherencia a la prevención
Posiblemente el concepto de adherencia preventiva no esté siendo contemplado como un constructo operativo en el ámbito de las adicciones, que puede determinar el éxito o fracaso en los resultados de la aplicación de programas. Se podría considerar, aunque sea únicamente en el plano hipotético, que cuando un programa cumple escrupulosamente con todos los requisitos necesarios para alcanzar unos resultados óptimos y no los consigue, ha sido como consecuencia de una baja adherencia a la prevención. Nos planteamos el objetivo de conceptualizar la adherencia en el contexto de la prevención de adicciones, con la intención de que se pueda utilizar como un referente más de la eficacia y eficiencia de los programas. Para ello habría que introducir en la evaluación de las intervenciones un sistema de medida de esta variable preventiva.

Hasta ahora el concepto de adherencia no se ha tenido en cuenta en el contexto de la prevención de las adicciones, posiblemente porque no se consideraba una variable que pudiera aportar información relevante o porque se ha asociado con otro tipo de comportamientos, fundamentalmente el de seguir las pautas farmacológicas de un tratamiento médico. En los últimos años se viene utilizando en contextos de actividad física y deporte, hablando de adherencia a la actividad deportiva en función de la inclusión y el mantenimiento del ejercicio físico en el estilo de vida de las personas (Márquez, Vives, y Garcés, 2010; Molinero, Salguero y Márquez, 2011; Ryan, Frederick, Lepes, Rubio, y Sheldon, 1997; Thogersen y Ntoumanis, 2006). En base a los usos que ha tenido del concepto de adherencia en otros ámbitos, consideramos que es necesario introducirlo en el marco de la prevención de adicciones, ya que puede actuar como predictor de éxito o fracaso en las intervenciones preventivas, entendiendo que a nivel teórico una persona adherente a un programa preventivo tiene más probabilidades de no ser consumidora.

\section{CONCEPTO DE ADHERENCIA}

A pesar de ser un término empleado ampliamente, sobre todo en el ámbito de la salud, sigue contando con algunas lagunas de interpretación, aplicación y consenso, solapándose en ocasiones con conceptualizaciones afines, que en esencia, podrían coincidir en contenido y significado. De hecho como señala Martín (2004, 2014), 
podemos encontrar en la literatura múltiples términos como cumplimiento, obediencia, seguimiento, adhesión, alianza terapéutica, colaboración, y cooperación que en última instancia podrían estar haciendo referencia al mismo constructo. En la actualidad, el término más utilizado es el de adherencia al tratamiento o adherencia terapéutica, y dado que el término se asocia al tratamiento y/o a la terapia, entendemos que sería fácilmente extrapolable a otros aspectos del comportamiento.

Alikari y Ziga (2014), hacen referencia a la multidimensionalidad del cumplimiento y cómo este relaciona la percepción del terapeuta y el paciente hacia el tratamiento a seguir, las cuales muchas veces no tienen por qué coincidir. Lo más novedoso es que se observa un cambio en la autonomía y participación en las decisiones terapéuticas del propio paciente, con el fin de que la adherencia pueda ser más alta.

Si revisamos alguna de las definiciones, comprobamos que Silva, Galeano y Correa (2005, p.269), entienden la adherencia como "el contexto en el cual el comportamiento de la persona coincide con las recomendaciones relacionadas con la salud e incluyen la capacidad del paciente para:

- Asistir a las consultas programadas (consultorio/hospital)

- Tomar los medicamentos como se prescribieron

- Realizar los cambios de estilo de vida recomendados

- Completar los análisis o pruebas solicitadas"

Esta definición se orienta a un conjunto de medidas farmacológicas y comportamentales que ha de observar el paciente para que la efectividad y eficiencia del tratamiento sea la más óptima.

Por su parte Martín (2014, p.229), define la adherencia al tratamiento como "un comportamiento complejo con carácter de proceso conformado por una estructura y dinámica interna, que integra un componente personal, un componente relacional en el que se encuentra implicado el profesional de salud y uno comportamental propiamente dicho, dirigidos al logro de un resultado beneficioso para la salud".

Como podemos comprobar por contraste entre ambas definiciones, seguimos en una conceptualización que no se establece como idéntica para todos los investigadores. La definición más difundida es la que formularon Sackett y Haynes (1976), en la que hacen una correspondencia entre el comportamiento del enfermo y las instrucciones clínicas y médicas recibidas. Por su parte Di Mateo y Di Nicola (1985), definen la adherencia terapéutica como "una implicación activa y voluntaria del paciente en el curso de un comportamiento aceptado de mutuo acuerdo con su médico con el fin de producir un resultado terapéutico deseado" (cit. Martín, 2014, p.227228). Esta sería una de las más admitidas, donde aparecen los términos de aceptación voluntaria y activa del enfermo para conseguir el resultado terapéutico recomendado y deseado.

Por otra parte, es fundamental tener en cuenta cuáles son los factores que impiden que una persona sea adherente, con el objetivo de poder determinar dónde radican los fallos del proceso y saber si parten del propio tratamiento, de quien lo recibe o de ambos. La noadherencia la podemos definir como el incumplimiento de las recomendaciones terapéuticas, sea de forma voluntaria o involuntaria (Silva et al., 2005). Los autores proponen cuatro factores intervinientes en el proceso de no-adherencia a la ingesta de medicamentos:

1. Prioridad que establece el paciente para la medicación

2. Percepción de gravedad del proceso de enfermedad

3. Nivel de credibilidad de la eficacia de los medicamentos

4. Aceptación por parte del paciente de los cambios recomendados

Según Ortiz y Ortiz (2007), la no-adherencia se puede generar a partir de una serie de factores:

- Personales: donde se podrían señalar la baja motivación, la inconsciencia o ignorancia del problema, la baja autoestima y autoeficacia, síntomas depresivos o depresión, entre otros

- Asistenciales: se demuestra que cuando la satisfacción del paciente es alta con el servicio médico o terapéutico aumenta la tasa de adherencia al tratamiento 
- Terapéuticos: se observan diferencias entre los tratamientos agudos y los crónicos. Asimismo a mayor complejidad en el tratamiento menor adherencia

Por último, habría que señalar también que el nivel de no-adherencia no es continuo ni estable. Una misma persona puede tomar una medicación y no otra o puede variar el cumplimiento a lo largo del tiempo en función de cómo percibe que funciona. La causa más probable de incumplimiento se atribuye al comportamiento de los sujetos y no al tratamiento en sí mismo.

\section{ADHERENCIA PREVENTIVA}

Hasta ahora los programas e implementaciones preventivas no han tenido en cuenta que una vez que se han expuesto los sujetos a un programa o intervención no tienen ningún tipo de supervisión directa por parte de los desarrolladores del programa sobre la población intervenida. Únicamente cuentan con el periodo o los periodos de seguimiento para evaluar hasta qué punto se han cubierto los objetivos a nivel de impacto, de proceso y de resultados, que son los que marcan el grado de eficacia y eficiencia del programa. La propuesta es introducir la adherencia a la prevención de las adicciones como una variable predictora más del éxito o fracaso de la intervención preventiva.

Se trata de saber previamente cuál es la intención de cumplimiento preventivo que tiene la población a intervenir. Posteriormente se puede calcular la tasa de cumplimiento real y establecer una relación entre ambas que nos permita mejorar las herramientas preventivas utilizadas. No podemos estimar el efecto de la prevención si no sabemos si la persona previamente intervenida cumple o no con las indicaciones preventivas.

Tal y como se ha expuesto para la adherencia y la noadherencia terapéutica, habría que introducir estrategias preventivas en el cuerpo de la implementación, que puedan aumentar la tasa de adherencia preventiva (Figura 1):

- Aumentar la motivación hacia el programa y la intervención

- Aumentar la percepción de riesgo
- Aumentar la credibilidad

- Confirmar la tasa de aceptación de cada una de las herramientas preventivas utilizadas

- Mejorar la inteligencia emocional

- Dirigir las actitudes hacia el mantenimiento de la salud

- Contrarrestar el efecto del grupo de iguales sobre la adherencia preventiva

- Configurar estrategias de apoyo familiar y social

- Reducir la complejidad de los contenidos preventivos

- Adaptar las intervenciones a las poblaciones

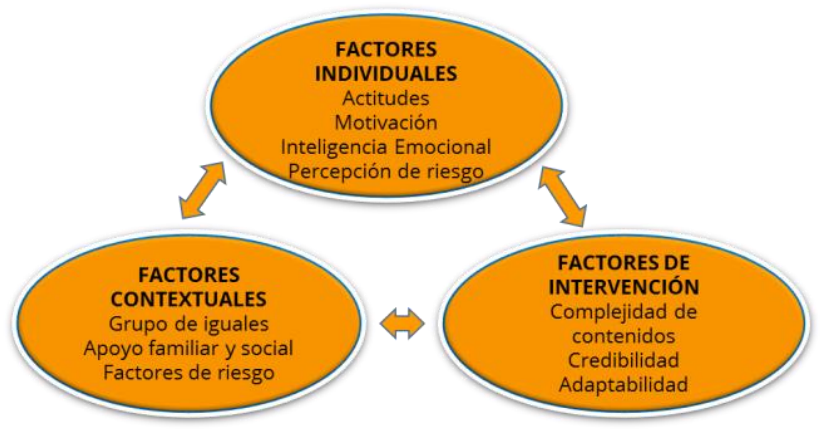

Figura 1. Determinantes de la adherencia preventiva en el ámbito de las adicciones (Modificado de Molinero et al., 2011)

A partir de los determinantes, podemos proponer la configuración teórica desde una perspectiva psicosocial de la adherencia preventiva, recogiendo aquellos elementos que estimamos que deben integrar este concepto, para poder introducirlo como una variable predictora en los modelos de intervención (Figura 2). 


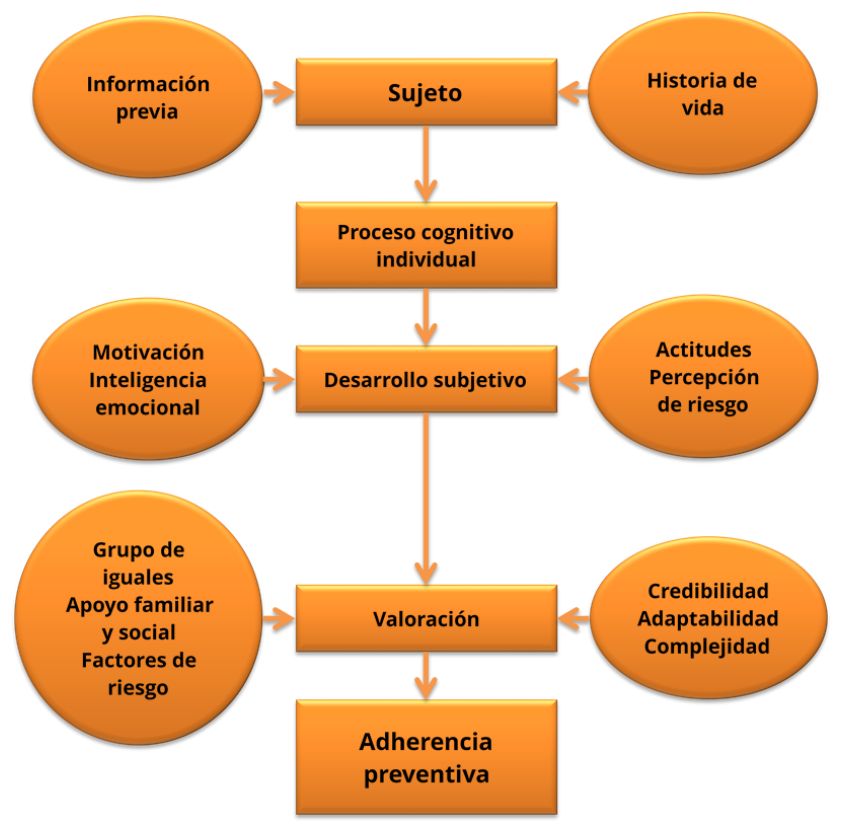

Figura 2. Configuración de la adherencia preventiva desde una perspectiva psicosocial

\section{El concepto de adherencia preventiva planteado desde algunos modelos psicosociales}

Como hemos podido observar, el concepto de adherencia puede estar relacionado con diferentes procesos psicológicos básicos, interaccionando con otras variables psicosociales. A partir de este supuesto teórico, podemos plantear la estructura de este concepto desde diferentes modelos y teorías psicosociales que de alguna forma han confirmado su influencia sobre el comportamiento de adherencia. De una forma ejemplar, se puede proponer el desarrollo de la adherencia preventiva desde la Teoría Social Cognitiva (Bandura, 1982, 2001) y el Modelo Transteórico (Prochaska y DiClemente, 1982, 1984).

Funcionamiento de la adherencia preventiva desde la Teoría Social Cognitiva

La teoría propone que las creencias hacen posible que las personas sean capaces de conseguir la meta comportamental deseada. La capacidad o habilidad para ejecutar un cambio de conducta y/o seguir las pautas preventivas informadas en un programa o intervención, están en función de la autoeficacia percibida. Esta creencia actuará como predictor de la conducta a seguir, interactuando con otros predictores.
En un planteamiento preventivo, una persona puede sentirse vulnerable y aumentar su percepción de riesgo ante un determinado comportamiento, pero podrá contar con la posibilidad de adoptar una conducta saludable previamente establecida por el programa y/o la intervención que haga disminuir su percepción de vulnerabilidad, pero si la persona tiene dudas sobre sus habilidades o aptitudes para llevar a cabo esa conducta saludable, la probabilidad de que la ejecute disminuye significativamente.

En función de este planteamiento cognitivo, se puede formular que la configuración de la adherencia preventiva se base en la interacción entre las variables del desarrollo subjetivo (motivación, inteligencia emocional, actitudes y percepción de riesgo) y la valoración que haga el sujeto de su vulnerabilidad percibida y su eficacia percibida. Esta interacción conseguirá que aumente o disminuya la adherencia preventiva (Figura 3).

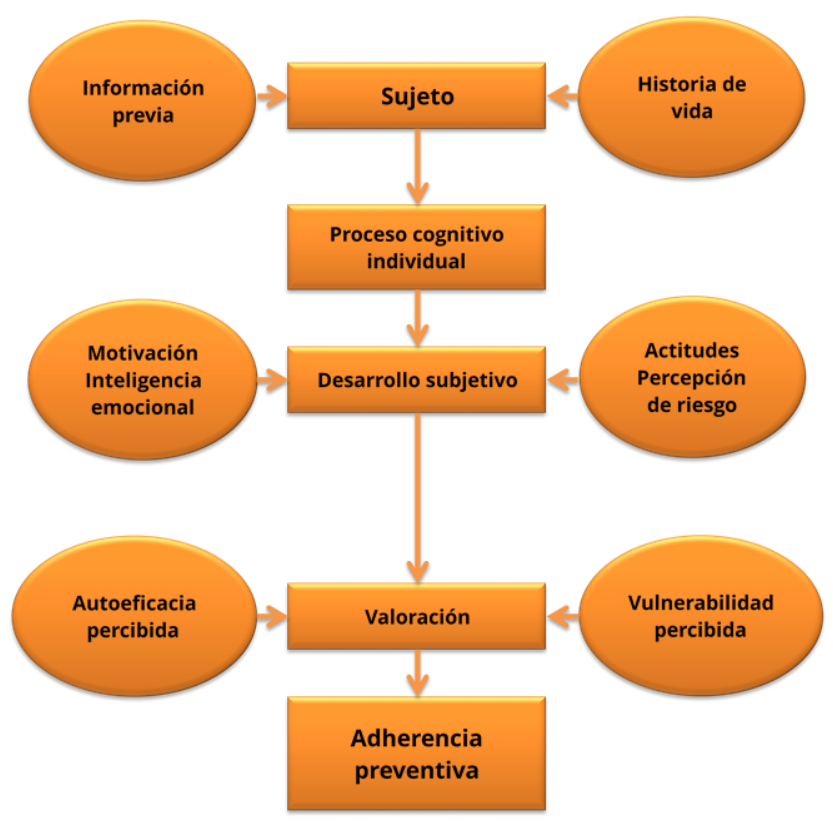

Figura 3. Funcionamiento de la adherencia preventiva desde la Teoría Social Cognitiva

Funcionamiento de la adherencia preventiva desde el Modelo Transteórico

Este modelo funciona de una forma ascendente mediante etapas de cambio posible, partiendo de un estadio donde la persona no es consciente del problema 
o situación. Conforme va tomando conciencia tendrá que ir superando el resto de las etapas, teniendo en cuenta que en el desarrollo del proceso interrelacionan otras variables que lo hacen posible o lo impiden.

Las etapas motivacionales que propone el modelo se pueden adaptar a la adherencia preventiva y sintetizar en las siguientes:

- Precontemplación: No existe intención ni voluntariedad en adquirir nuevos comportamientos o llevar a cabo cambios. No se cuenta con suficiente información o no se tiene conciencia de que exista o pueda existir en el futuro un problema si se adoptan determinados comportamientos de riesgo.

- Contemplación: Se cuenta con suficiente información, pero no existe voluntariedad de ejecutar determinados comportamientos saludables o cambiar aquellos que puedan ser peligrosos.

- Preparación: Se dan algunos pequeños pasos hacia los comportamientos saludables. Se empieza a ser más consciente de la necesidad de correr menos riesgos.

- Acción: Esta es la etapa crucial en la que se afianzan los comportamientos saludables frente a los de riesgo.

- Mantenimiento: En esta última etapa se consolidan los comportamientos saludables y se mantienen a lo largo del tiempo.

Para la configuración de la adherencia preventiva, habría que contar además con los procesos de adquisición o cambio de comportamientos (Prochaska y Velicer, 1997), que ejercen de motores en la adquisición o cambio de comportamientos, entre los que se pueden incluir el control de estímulos, la autoevaluación o el apoyo social y familiar. Finalmente, en la fase valorativa de la configuración de la adherencia preventiva tendremos que tener en cuenta la autoeficacia percibida, los procesos de decisión, las emociones positivas y/o negativas y la posible pérdida de control de impulsos (Figura 4).

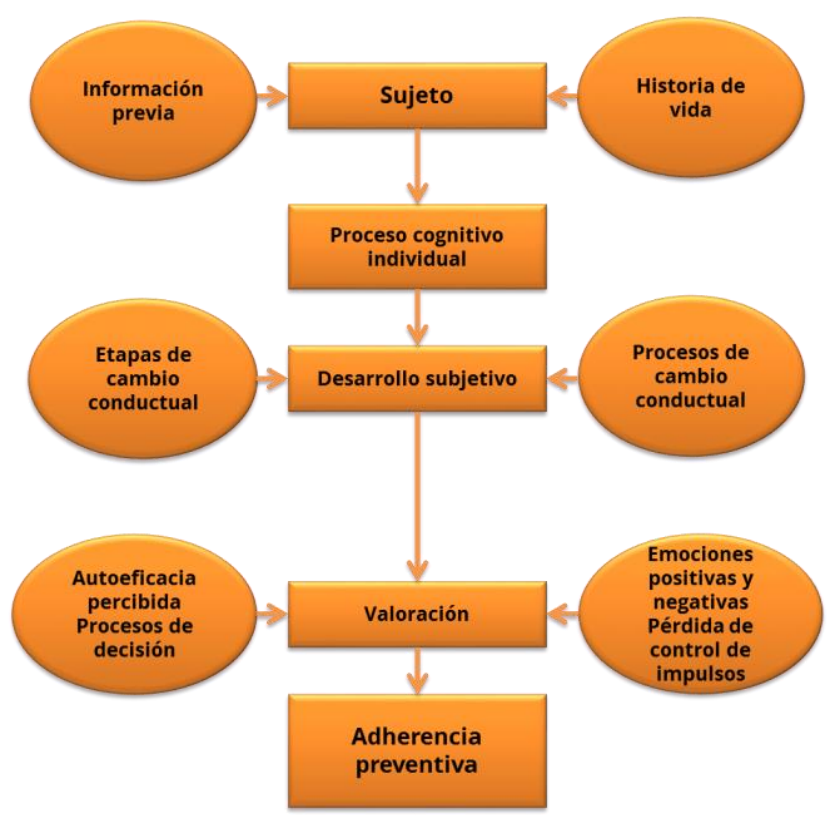

Figura 4. Funcionamiento de la adherencia preventiva desde el Modelo Transteórico

\section{REFLEXIONES FINALES}

El concepto de adherencia se encuentra entre los constructos mal definidos, pero es necesario rescatarlo y perfilarlo adecuadamente ya que su utilidad es de gran importancia, tanto en el plano terapéutico como en el de promoción y prevención de salud. La adherencia terapéutica tiene la ventaja implícita de que la persona que acude solicitando ayuda médica o psicológica se encuentra con una necesidad imperiosa de mejorar su estado de salud. Su funcionamiento dependerá del grado de necesidad en el que se encuentre el paciente en cada caso pero, en líneas generales, la probabilidad de ser adherente, obediente o cumplidor es más alta potencialmente.

Como señalan acertadamente Ortiz y Ortiz (2007), las recomendaciones terapéuticas de cualquier índole no pueden tener un seguimiento o supervisión directa cotidianamente a través de un agente externo. Se hace imprescindible transferir la responsabilidad del seguimiento al propio paciente que bajo su responsabilidad tendrá que hacer fiel cumplimiento de las prescripciones para intentar resolver el problema de salud.

El concepto de adherencia preventiva incluiría dos propuestas concretas, una enmarcada en el plano 
intencional, que nos permitiera inferir la cantidad de cumplimiento que está dispuesto a asumir la persona intervenida con las propuestas y estrategias preventivas que se le proporcionan, y otra desde los resultados obtenidos en el seguimiento de los programas, que incluyan esta variable para calcular la cantidad de varianza que podrían explicar del comportamiento de salud.

La relevancia que adquiere el concepto de adherencia preventiva, se encuentra en la línea que plantean Molinero et al. (2011), pero más orientada hacia comportamientos de mantenimiento de la salud. Habría que basar la importancia de la adherencia en varios preceptos:

- Ausencia de programas de prevención de adicciones que consideren esta variable.

- Tasas de credibilidad de las estrategias preventivas bajas, que redundan en una adherencia débil.

- Propuestas poco realistas para favorecer los cambios en el estilo de vida de la población diana de los programas preventivos.

- Baja percepción de riesgo al consumo que colisiona frontalmente con la propuesta de adherencia preventiva.

Se puede intentar aumentar la adherencia preventiva utilizando evidencias de otras investigaciones. Si aumentamos las creencias de control, la autoeficacia percibida y la capacidad de autorregulación, tendremos una mayor probabilidad de que aumente la adherencia (Sherman y Koelmeyer, 2012). Basándonos en las apreciaciones de Jiménez (2014), si aumentamos la motivación y centramos los programas en la modificación de comportamientos de riesgo, mejora del estilo de vida y satisfacción de necesidades incentivando la participación activa de la población diana, tendremos más probabilidad de conseguir una conducta adherente a la prevención. Además, en otras investigaciones se ha señalado que la adherencia a la actividad física aumenta cuando la población intervenida presenta tasas mayores de autoeficacia y autocontrol (Robinson, Newton, Jones y Dawson, 2014), la credibilidad de la información suministrada aumenta la tasa de adherencia (Gustafsson, Wettermark, Godman, Andersén-Karlsson, Bergman,
Hasselstrçm, Hensjç et al., 2011), o que cuando aumenta el optimismo disposicional se da una menor tasa de adherencia en adictos a la heroína (Zaidi, 2014).

Finalmente, habría que hacer un esfuerzo por incorporar esta variable a la evaluación de los programas e intervenciones preventivas, con el fin de poder ser más objetivos a la hora de cuantificar los resultados finales de las aplicaciones. La evaluación preventiva no estará completa si no tenemos una mayor certeza de que los cambios de comportamiento, si los hay, se pueden atribuir al programa aplicado, y para ello necesitamos saber si la población intervenida ha cumplido con las recomendaciones planteadas en el programa o la intervención, es decir, si ha sido adherente.

\section{REFERENCIAS}

Alikari, V. y Ziga, S. (2014). Conceptual analysis of patient compliance in treatment. Health Science Journal, 8(2), 179-186.

Ariza, C., Villalbí, J. R., Sánchez-Martínez, F. y Nebot, M. (2011). La evaluación del proceso en relación con la evaluación de la efectividad: experiencias de programas en el medio escolar. Gaceta Sanitaria, 25, 32-39.

Bandura, A. (1982). Self-efficacy mechanism in human agency. American Psychology, 37, 122-47.

Bandura, A. (2001). Social Cognitive Theory: An Agentic Perspective. Annual Review Psychology, 52, 1-26.

Becoña, E. (1999). Bases teóricas que sustentan los programas de prevención de drogas. Madrid: Plan Nacional sobre Drogas.

Bejarano, J., Ahumada, G., Sánchez, G., Nora, M., Hynes, M. y Cumsille, F. (2011). Perception of Risk and Drug Use: An Exploratory Analysis of Explanatory Factors in Six Latin American Countries. The Journal of International Drug, Alcohol and Tobacco Research, 1(1), 9-17.

Boustani, M.M., Frazier, S.T., Becker, K.D., Bechor, M., Dinizulu, S.M., Hedemann, E.R., Ogle, R.R. y Pasalich D.S. (2014). Common Elements of Adolescent Prevention Programs: Minimizing Burden While Maximizing Reach. Administration and Policy in Mental Health and Mental Health Services Research, 41(192), 1-11.

Di Matteo R. y Di Nicola D. (1985). Achieving Patient Compliance. The Psychology of the Medical Practitioner's Role. New York: Pergamon.

García del Castillo, J.A. (2011). La evaluación del consumo de drogas en perspectiva. Health and Addictions/Salud y Drogas, 11(1), 7-15.

García del Castillo, J.A. (2012). Concepto de percepción de riesgo y su repercusión en las adicciones. Health and Addictions/Salud y Drogas, 12(2), 133-151. 
García del Castillo, J.A., López-Sánchez, C. y García del Castillo-López, A. (2014). La percepción de riesgo en la prevención del consumo de alcohol. Bilbao: Universidad de Deusto.

García del Castillo, J.A., López-Sánchez, C., García del Castillo-López, A. y Dias, P. (2014). Análisis de la información en la prevención del consumo de drogas y otras adicciones. Health and Addictions/Salud y Drogas, 14(1), 5-14.

García del Castillo-López, A., García del Castillo, J.A. y Marzo, J.C. (2012). La relevancia de la inteligencia emocional en la prevención del consumo de alcohol. Informacio Psicológica, 104, 100-111.

Gázquez, M., García del Castillo, J.A. y Espada, J.P. (2011). Eficacia diferencial de dos programas de prevención escolar sobre el consumo de tabaco, según el tipo de aplicador. Psicothema, 23(4), 537-543.

Gázquez, M., García del Castillo, J.A. y Ruiz, I. (2011). Importancia de la fidelidad en la implementación de programas escolares para prevenir el consumo de drogas. Health and Addictions/Salud $y$ Drogas, 11(1), 51-69.

Gil, E., González, J.R. y Meneses, C. (2010). Percepción del riesgo y dinámicas promotoras de salud en adolescentes: una mirada de género. Revista Española de Drogodependencias, 35(3), 297-308.

Grant, J. E., Potenza, M. N., Weinstein, A. y Gorelick, D. A. (2010). Introduction to behavioral addictions. The American Journal of Drug and Alcohol Abuse, 36(5), 233-241.

Gustafsson, L.L., Wettermark, B., Godman, B., Andersén-Karlsson, E., Bergman, U., Hasselstrçm, J., Hensjç, L. et al. (2011). The 'Wise List' A Comprehensive Concept to Select, Communicate and Achieve Adherence to Recommendations of Essential Drugs in Ambulatory Care in Stockholm. Basic \& Clinical Pharmacology \& Toxicology, 108, 224-233.

Holder, H. (2009). Prevention programs in the 21st century: what we do not discuss in public. Addiction, 105(4), 578-581.

Jiménez, L. (2014). Adherencia terapéutica y oportunidades de mejora del estado salud-enfermedad. Revista Costa Rica de Salud Pública, 23, 6874

Jiménez, L., Antolín-Suárez, L., Oliva, A., Hidalgo, V., Jiménez-lglesias, A., Lorence, B., Moreno, C. y Ramos, P. (2014). A synthesis of research on effectiveness of school-based drug prevention programmes/Claves de eficacia de los programas de prevención de consumo de sustancias en el contexto escolar: una meta-revisión. Cultura y Educación, (ahead-of-print), 1-30.

Londoño, C. y Vinaccia, S. (2005). Prevención del abuso en el consumo de alcohol en jóvenes universitarios: lineamientos en el diseño de programas costo-efectivos. Psicología y Salud, 15(2), 241-249.

Márquez, S., Vives, L. y Garcés de los Fayos, E. (2010). Adherencia y abandono en la actividad física y deportiva. En S. Márquez y N. Garatachea (Eds.). Actividad Física y Salud (pp. 225-239). Madrid: Díaz de Santos.

Martín, L. (2004). Acerca del concepto de adherencia terapéutica. Revista Cubana de Salud Pública, 30(4). En línea. Recuperado el 02 de diciembre

de

2014:

http://scielo.sld.cu/scielo.php?script=sci arttext\&pid=S086434662004000400008

Martín, L. (2014). Marco conceptual para la evaluación y mejora de la adherencia a los tratamientos médicos en enfermedades crónicas. Revista Cubana de Salud Pública, 40(2), 225-238.

Molinero, O.; Salguero del Valle, A.; Márquez, S. (2011). Autodeterminación y adherencia al ejercicio: estado de la cuestión. Revista Internacional de Ciencias del Deporte, 25(7), 287-304.

Olivar, A. (2011). Aplicaciones de la neurociencia de las adicciones en los modelos preventivos. Trastornos Adictivos, 13(1), 20-26.

Ordoñana, J.R. y Gómez, J. (2002). Amenaza y persuasión en mensajes de salud sobre consume de alcohol: de la teoría a la práctica. Adicciones, 14(4), 479-485.

Ortiz, M. y Ortiz, E. (2007). Psicología de la salud: Una clave para comprender el fenómeno de la adherencia terapéutica. Revista Médica de Chile, 135, 647-652.

Pons, J. (2008). Modelos interpretativos del consumo de drogas. Polis, 4(2), 157-186

Prochaska, J. y DiClemente, C. (1982). Transtheoretical therapy: Toward a more integrative model of change. Psychother Theory Res Pract, 19, 276-88.

Prochaska, J. y DiClemente, C. (1984). The transtheoretical approach. New York: Dow Jones.

Prochaska, J. y Velicer, W. (1997). The transtheore tical model of health behavior change. American Journal of Health Promotion, 12, 38-48.

Rendón, M.A. (2005). Relación entre los conceptos: información, conocimiento y valor. Semejanzas y diferencias. Ciencias de la Información, Brasília, 34(2), 52-61.

Ryan, R. M., Frederick, C. M., Lepes, D. D., Rubio, N. y Sheldon, K. M. (1997). Intrinsic motivation and exercise adherence. International Journal of Sport Psychology, 28, 335-354.

Sackett, D.L. y Haynes, B. (1976). Compliance with Therapeutic Regimens. Baltimore: Johns Hopkins University Press.

Sherman, K.A. y Koelmeyer, L. (2012). Psychosocial predictors of adherence to lymphedema risk minimization guidelines among women with breast cáncer. Psycho-Oncology, 22(5), 1120-1126.

Silva, G.E., Galeano, E. y Correa, J.O. (2005). Adherencia al tratamiento. Implicaciones de la no-adherencia. Acta Médica Colombiana, 30(4), 268-273.

Solinas, M., Thiriet, N., Chauvet, C. y Jaber, M. (2010). Prevention and treatment of drug addiction by environmental enrichment. Progress in neurobiology, 92(4), 572-592.

Thogersen, C. y Ntoumanis, N. (2006). The role of self-determined motivation to the understanding of exercise-related behaviours, cognitions and physical self-evaluation. Journal of Sport Sciences, 24, 393-404. 
Uribe, J.I., Verdugo, J.C. y Salinas, X.Z. (2011). Relación entre percepción de riesgo y consumo de drogas en estudiantes de bachillerato. Psicología y Salud, 21(1), 47-55.

Valentine, J. y Dejong, J. (2013). Substance Abuse Prevention in Multicultural Communities. N.Y.: Routledge.

Ying, M.A., Jisoo, A.H.N., Lewis, N. y Martínez, L. (2014). Are drug prevention efforts fulfilling the informational needs of college students? A survey of engagement with information about topics related to marijuana and amphetamines from media, medical and interpersonal sources. 142nd APHA Annual Meeting and Exposition. New Orleans.

Zaidi, U. (2014). Dispositional Optimism and Treatment Compliance in Heroin Addicts. Journal of Humanities And Social Science, 19(10), 125131.

Zimmermann, G. (2010). Risk perception, emotion regulation and impulsivity as predictors of risk behaviours among adolescents in Switzerland. Journal of Youth Studies 13(1), 83-99. 
